\title{
An algebraic condition and an algorithm for the internal contact between two ellipsoids
}

\author{
Ibrahim Trabelsi and Maher Moakher \\ National Engineering School at Tunis, LAMSIN-ENIT \& FS Gabés \\ Tunis-Belvédère, Tunisia, and \\ Cendrine Gatumel and Henri Berthiaux \\ Ecoles des Mines d'Albi, Centre RAPSODEE-EMAC, UMR CNRS 2392, \\ Albi, France
}

\begin{abstract}
Purpose - The purpose of this paper is to present a new method for the detection and resolution of the contact point between two ellipsoids. Numerical simulations of ellipsoidal particles in a rotary cylinder are also presented.

Design/methodology/approach - An algebraic condition is developed for the internal contact between two ellipsoids and an efficient contact detection algorithm for overlapping ellipsoids is implemented.

Findings - This method was found to have the advantages of effectiveness and speed in the detection and resolution of the contact point.

Originality/value - The dynamics of granular materials are of great importance in many industries dealing with powders and grains, such as pharmaceutical, chemical, and food industries. The main difficulty of such simulations is the excessive CPU time required for a large number of particles. In the discrete element method, contact detection between grains is the most expensive step in solving a nonlinear system for determination of the contact point, the normal vector and the overlap distance between ellipsoids. The numerical behavior and the optimization of the new algorithm presented in this paper are important also.
\end{abstract}

Keywords Programming and algorithmic languages, Geometric planes and solids

Paper type Research paper

\section{Introduction}

The problem of contact detection between two objects arises in various applications such as robotics, geomechanics, computer graphics, computer animation, etc. In the discrete element simulation of the flow of granular materials, one also needs to resolve the contact, i.e. to determine the "contact point" and "contact normal direction". The problems of detection and resolution of contact are sometimes coupled in many algorithms. However, the resolution of the contact can be important in terms of computing time. It is therefore desirable to devise an algorithm that separates these two steps so that when there is no contact the second step is not performed.

Several studies are interested in the problem of the intersection of two quadratic surfaces in three-dimensional space. Some mathematical tools are used to resolve this problem. Segre's characteristics is used to classify the intersection curves between two quadric surfaces. Descartes' rule of signs, first described by Réné Descartes, is a technique for determining the number of positive or negative roots of a polynomial. Sturm theorem makes it possible to calculate the number of distinct real roots, belonging to a given interval, of a polynomial function.

Among the works that are interested only on the contact detection we cite: (Bromwich, 1906; Sommerville, 1947) for the classification of quadratic forms, (Farouki 
et al., 1989; Levin, 1979) for the structure and parameterization of the intersection curve, and (Perram and Wertheim, 1985; Perram et al., 1996) for molecular dynamics simulations. Among the works interested in detecting and resolving the contact point problem we mention (Lin and Ng, 1995; Trabelsi and Moakher, 2006) for discrete element simulations, and (Wang et al., 2001) for the algebraic condition of external contact between ellipsoids.

Ellipsoids have a relatively simple convex form that can approximate the shape of several nature and man-made grains. This justifies the needs for developing an efficient method for the resolution of contact between two overlapping ellipsoids. In this paper we present an algebraic condition for the internal contact and a new algorithm for the determination of the contact point and the overlapping distance of two ellipsoids. The determination of the signs of the roots of the characteristic equation of two ellipsoids allows the contact detection. Indeed, the roots of the characteristic equation changes sign depending on the state of two ellipsoids: separation, inclusion or overlapping. Our work is an extension of (Wang et al., 2001) for the algebraic condition of internal contact, and of (Lin and Ng, 1995; Trabelsi and Moakher, 2006) for the efficient algorithm of contact detection.

The organization of this paper is as follows. In section 2, we review the algebraic conditions for the internal intersection of a pair of ellipsoids. In section 3, we present an iterative procedure that efficiently solve the contact problem based on the algebraic condition and the concept of geometric potential. In section 4, we present simulation results for ellipsoidal particles inside a rotary cylinder.

\section{Intersection of two ellipsoids}

Definition 2.1: Given two ellipsoids $\mathcal{A}$ and $\mathcal{B}$ we say that:

- $\mathcal{B}$ is included in $\mathcal{A}$ if $\mathcal{A} \cap \mathcal{B}=\mathcal{B}$ (See Figure 1(a)).

- $\mathcal{A}$ and $\mathcal{B}$ are tangent internally if $\mathcal{A} \cap \mathcal{B}=\mathcal{A}$ or $\mathcal{B}$ and their external surfaces have only one common point (See Figure 1(b)).

- $\mathcal{A}$ and $\mathcal{B}$ are overlapping if their external surfaces have common points (See Figure 1(c)).

Figure 1.

Inclusion, internal tangency and overlap of two ellipsoids

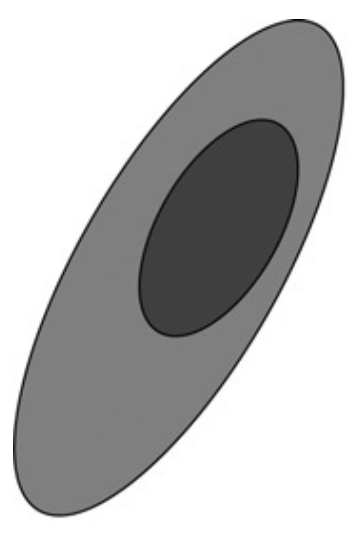

(a)

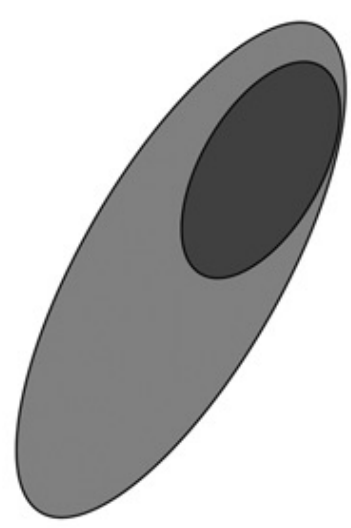

(b)

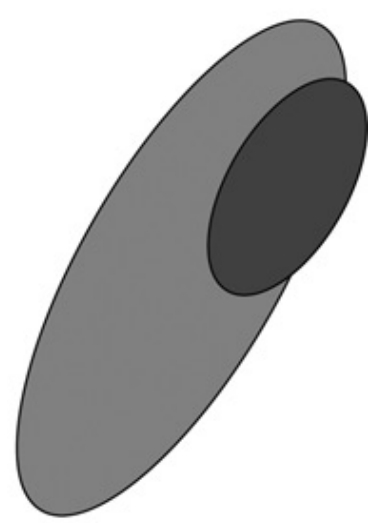

(c) 
Let $\mathcal{A}$ and $\mathcal{B}$ be two ellipsoids in three-dimensional space. To $\mathcal{A}$ we associate the $4 \times 4$ symmetric and real matrix $A$ such that the boundary of $\mathcal{A}$ is characterized by the equation $\boldsymbol{x}^{T} A \boldsymbol{x}=0$, where $\boldsymbol{x}=(x, y, z, 1)$ represents homogeneous coordinates. Similarly, to $\mathcal{B}$ we associate the $4 \times 4$ symmetric and real matrix $B$ such that the boundary of $\mathcal{B}$ is characterized by the equation $\boldsymbol{x}^{T} B \boldsymbol{x}=0$.

\subsection{Algebraic condition}

To the pair $\{\mathcal{A}, \mathcal{B}\}$ of ellipsoids, we introduce the function $h(\lambda)=\operatorname{det}(\lambda A+B)$ which is a polynomial of degree 4 in $\lambda$. The equation $h(\lambda)=0$ is called the characteristic equation of $\mathcal{A}$ and $\mathcal{B}$.

The goal of this work is to show the following results:

- $\mathcal{A} \cap \mathcal{B}=\mathcal{A}$ or $\mathcal{B}$ if and only if the characteristic equation $h(\lambda)=0$ has four negative roots.

- $\mathcal{A}$ and $\mathcal{B}$ touch each other internally at a single point if and only if characteristic equation $h(\lambda)=0$ has a negative double root.

Theorem 2.2: $\quad$ The characteristic equation $h(\lambda)=0$ has at least two negative roots.

Proof: $\quad$ There exists an affine transformation $P$ which transforms $\mathcal{A}$ in its standard form $\mathcal{A}^{*}$ and $\mathcal{B}$ into a sphere $\mathcal{B}^{*}$ :

$$
\begin{aligned}
& \mathcal{A}^{*}: \boldsymbol{x}^{T} A^{*} \boldsymbol{x}=\boldsymbol{x}^{T} P^{T} A P \boldsymbol{x}=0, \\
& \mathcal{B}^{*}: \boldsymbol{x}^{T} B^{*} \boldsymbol{x}=\boldsymbol{x}^{T} P^{T} B P \boldsymbol{x}=0,
\end{aligned}
$$

where $A^{*}$ and $B^{*}$ are the corresponding matrices which are given by

$$
A^{*}=\left\{\begin{array}{cccc}
1 / a^{2} & & & \\
& 1 / b^{2} & & \\
& & 1 / c^{2} & \\
& & & -1
\end{array}\right\}, B^{*}=\left\{\begin{array}{cccc}
1 & & & -x_{0} \\
& 1 & -y_{0} \\
& & 1 & -z_{0} \\
-x_{0} & -y_{0} & -z_{0} & -r^{2}+x_{0}^{2}+y_{0}^{2}+z_{0}^{2}
\end{array}\right\} .
$$

We note that $\operatorname{det}\left(\lambda A^{*}+B^{*}\right)=\operatorname{det}(\lambda A+B) \cdot\left(\operatorname{det}\left(P^{-1}\right)\right)^{2}=0$. Therefore, although the characteristic equation of $\mathcal{A}$ and $\mathcal{B}$ is different from the characteristic equation of $\mathcal{A}^{*}$ and $\mathcal{B}^{*}$, both equations have the same roots. Thus, the problem of finding the roots of the characteristic equation $h(\lambda)=0$ of the two ellipsoids $\mathcal{A}$ and $\mathcal{B}$ is equivalent to the problem of finding the roots of the characteristic equation $h^{*}(\lambda)=0$ of the two ellipsoids $\mathcal{A}^{*}$ and $\mathcal{B}^{*}$. The latter equation writes

$$
\begin{aligned}
h(\lambda)= & -\left(\frac{\lambda}{a^{2}}+1\right)\left(\frac{\lambda}{b^{2}}+1\right)\left(\frac{\lambda}{c^{2}}+1\right)\left(\lambda+r^{2}\right)+\lambda\left(\frac{\lambda}{b^{2}}+1\right)\left(\frac{\lambda}{c^{2}}+1\right)\left(\frac{x_{0}^{2}}{a^{2}}\right) \\
& +\lambda\left(\frac{\lambda}{a^{2}}+1\right)\left(\frac{\lambda}{c^{2}}+1\right)\left(\frac{y_{0}^{2}}{b^{2}}\right)+\lambda\left(\frac{\lambda}{a^{2}}+1\right)\left(\frac{\lambda}{b^{2}}+1\right)\left(\frac{z_{0}^{2}}{c^{2}}\right) .
\end{aligned}
$$

Without loss of generality we assume that $0<a \leq b \leq c$. Then it is easy to check that $h\left(-a^{2}\right) h\left(-b^{2}\right) \leq 0$ and $h\left(-b^{2}\right) h\left(-c^{2}\right) \leq 0$. By the intermediate value theorem it follows that $h(\lambda)=0$ has at least two negative roots in $\left[-c^{2},-a^{2}\right]$. 
Lemma 2.3: If two ellipsoids $\mathcal{A}$ and $\mathcal{B}$ touch each other internally at a single point, then their characteristic equation $h(\lambda)=0$ has a negative double root.

Proof: Assume that $\mathcal{A} \cap \mathcal{B}=\mathcal{B}$ and $X_{0}^{t} A X_{0}=X_{0}^{t} B X_{0}=0$, where $X_{0}$ is the intersection point between $\mathcal{A}$ and $\mathcal{B}$. If $\mathcal{A}$ and $\mathcal{B}$ have the same tangent plane at $X_{0}$ then $B X_{0}=-\lambda_{0} A X_{0}$ for some real value $\lambda_{0} \neq 0$. Thus, $\left(\lambda_{0} A+B\right) X_{0}=0$ and $\lambda_{0}$ is a root of $h(\lambda)=0$. We take $U_{0}$ a point such that $U_{0}^{t} A U_{0}=0$ and $V_{0}$ the intersection of the line passing by $X_{0}$ and $U_{0}$ and the external surface of $\mathcal{B}$. We have $V_{0}^{t} B V_{0}=0$. Let $Y_{0}$ be a point on the line passing by $X_{0}$ and $U_{0}$ and is inside $\mathcal{B}$ and hence inside $\mathcal{A}$. Then $Y_{0}^{t} B Y_{0}<0$ and $Y_{0}^{t} A Y_{0}<0$. By construction we have

$$
\begin{aligned}
& U_{0}=s Y_{0}+(1-s) X_{0} ; s>1 \text { since } Y_{0} \text { is in } \mathcal{A} \\
& V_{0}=r Y_{0}+(1-r) X_{0} ; r>1 \text { since } Y_{0} \text { is in } \mathcal{B}
\end{aligned}
$$

Therefore,

$$
\begin{aligned}
0=U_{0}^{t} A U_{0} & =\left((1-s) X_{0}+s Y_{0}\right)^{t} A\left((1-s) X_{0}+s Y_{0}\right) \\
& =\left((1-s) X_{0}^{t}+s Y_{0}^{t}\right) A\left((1-s) X_{0}+s Y_{0}\right) \\
& =(1-s)^{2} X_{0}^{t} A X_{0}+s^{2} Y_{0}^{t} A Y_{0}+2 s(1-s) Y_{0}^{t} A X_{0} ; X_{0}^{t} A X_{0}=0 \\
& =s^{2} Y_{0}^{t} A Y_{0}+2 s(1-s) Y_{0}^{t} A X_{0},
\end{aligned}
$$

and hence

$$
Y_{0}^{t} A X_{0}=\frac{s}{2(s-1)} Y_{0}^{t} A Y_{0}<0 .
$$

Similarly, from $V_{0}^{t} B V_{0}=0$ we obtain

$$
Y_{0}^{t} B X_{0}=\frac{r}{2(r-1)} Y_{0}^{t} B Y_{0}<0
$$

As $\left(\lambda_{0} A+B\right) X_{0}=0$, we have $Y_{0}^{t}\left(\lambda_{0} A+B\right) X_{0}=\lambda_{0} Y_{0}^{t} A X_{0}+Y_{0}^{t} B X_{0}=0$, which implies that $\lambda_{0}=-\frac{Y_{0}^{t} B X_{0}}{Y_{0}^{t} A X_{0}}<0$. The product of the four roots of $h(\lambda)=0$ is $(a b c r)^{2}>0$, $h(\lambda)=0$ have two negative roots, $\lambda_{0}<0$ then the fourth root $\lambda_{1}$ must be negative. It remains to prove that $\lambda_{0}=\lambda_{1}$.

Suppose $\lambda_{0} \neq \lambda 1$, then $X_{1} \neq X_{0}$ is the eigenvector associated with $\lambda_{1}$. Then $\left(\lambda_{0} A+B\right) X_{0}=0,\left(\lambda_{1} A+B\right) X_{1}=0$ and $X_{1}^{t}\left(\lambda_{0} A+B\right) X_{0}=0, X_{0}^{t}\left(\lambda_{1} A+B\right) X_{1}=0$. We have $X_{1}^{t} B X_{0}=X_{1}^{t} A X_{0}=X_{0}^{t} B X_{1}=X_{0}^{t} A X_{1}=0$, which implies that $X_{1}$ is a common point on the tangent plane of $\mathcal{A}$ and $\mathcal{B}$ and $X_{1}$ is outside $\mathcal{A}$ and $\mathcal{B}$. It follows that $X_{1}^{t} A X_{1}=X_{1}^{t} B X_{1}>0$ and $X_{1}^{t}\left(\lambda_{1} A+B\right) X_{1}>0$. This is a contradiction since $\left(\lambda_{1} A+B\right) X_{1}=0$.

Lemma 2.4: If the characteristic equation $h(\lambda)=0$ has a negative double root, then the two ellipsoids $\mathcal{A}$ and $\mathcal{B}$ touch each other internally at a single point.

Proof: Let $\lambda_{0} \neq 0$ be negative double root of $h(\lambda)=0$ and let $X_{0}$ be the associated eigenvector. Then $\lambda_{0}$ is also an eigenvalue of $-A^{-1} B$ with multiplicity 2 . Let $X_{1}$ be the generalized eigenvector defined by $\left(\lambda_{0} I-A^{-1} B\right)^{2} X_{1}=0=\left(\lambda_{0} I-A^{-1} B\right)$ 
$X_{0}$. It follows that $\left(\lambda_{0} I-A^{-1} B\right) X_{1}=X_{0}$. Since $A$ and $B$ are symmetric we have $X_{0}^{t} A X_{0}=\left(\left(\lambda_{0} I-A^{-1} B\right) X_{1}\right)^{t} A\left(\lambda_{0} I-A^{-1} B\right) X_{1}=X_{1}^{t} A\left(\lambda_{0} I-A^{-1} B\right)^{2} X_{1}=0$. $X_{0}^{t} B X_{0}=\left(\left(\lambda_{0} I-A^{-1} B\right) X_{1}\right)^{t} B\left(\lambda_{0} I-A^{-1} B\right) X_{1}=X_{1}^{t} B\left(\lambda_{0} I-A^{-1} B\right)^{2} X_{1}=0$. Then $X_{0}$ is a point of both $\mathcal{A}$ and $\mathcal{B}$. As $X_{0}$ is the eigenvector associated with $\lambda_{0}$, we have $h\left(\lambda_{0}\right)=\left(\lambda_{0} A+B\right) X_{0}=0$. Thus, the tangent plane of $\mathcal{A}$ and $\mathcal{B}$ at $X_{0}$ are $X^{t} A X_{0}=X^{t} B X_{0}=0$ are the same. We therefore conclude that $\mathcal{A}$ and $\mathcal{B}$ are tangent at $X_{0}$.

Lemma 2.5: If the external surfaces of $\mathcal{A}$ and $\mathcal{B}$ have common points, then $h(\lambda)=0$ does not have four negative roots.

Proof: It has been proved that $h(\lambda)=0$ has a double positive roots if $\mathcal{A}$ and $\mathcal{B}$ are tangent externally (Wang et al., 2001) and has double negative roots if $\mathcal{A}$ and $\mathcal{B}$ are tangent internally, cf. Lemma 2.4. Note that the case where there is intersection between the two ellipsoids is in between these two situations (Figure 2). Therefore, two of the roots change from positive into negative through 0 or $\infty$, without ever becoming imaginary roots, which is a contradiction by the continuity of the solution (Bhatia, 1997) and since the leading coefficient of $h(\lambda)=0$ is $-(a b c)^{-2} \neq 0$.

Lemma 2.6: If $\mathcal{A} \cap \mathcal{B}=\mathcal{A}$ or $\mathcal{B}$, then $h(\lambda)=0$ has four negative roots.

Proof: If $\mathcal{A} \cap \mathcal{B}=\mathcal{A}$ or $\mathcal{B}$, then the two ellipsoids are either:

(1) tangent internally;

(2) or one is included in the other.

It has been proved that if the two ellipsoids $\mathcal{A}$ and $\mathcal{B}$ are tangent internally then $h(\lambda)=0$ has a double negative roots, which implies that $h(\lambda)=0$ has four negative roots by Theorem 2.2 .

If $\mathcal{A} \cap \mathcal{B}=\mathcal{A}$ or $\mathcal{B}$ and the two ellipsoids $\mathcal{A}$ and $\mathcal{B}$ are not tangent internally, then $\mathcal{A}$ is included in $\mathcal{B}$, or $\mathcal{B}$ is included in $\mathcal{A}$. Let us assume that $\mathcal{B}$ is included in $\mathcal{A}$. If $\mathcal{A}$ and $\mathcal{B}$ have the same center $\left(x_{0}=y_{0}=z_{0}=0\right)$, then $h(\lambda)=0$ have four negative roots

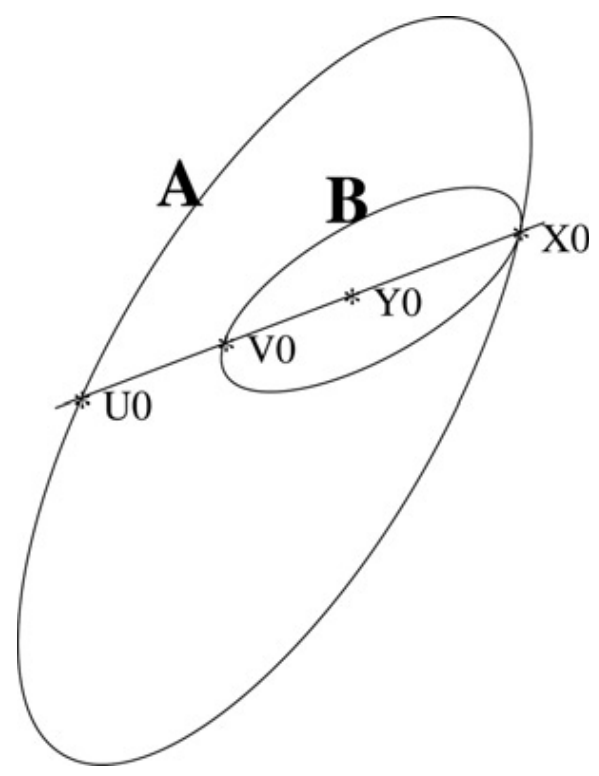

Figure 2. Two ellipsoids $\mathcal{A}$ and $\mathcal{B}$ touching each other internally at a single point $X_{0}$ 
$\left(-a^{2},-b^{2},-c^{2},-r^{2}\right)$. Let $\mathcal{B}(t)$ be a family of ellipsoids that represents a continuous motion from $\mathcal{B}\left(t_{0}\right)=\mathcal{B}$ to $\mathcal{B}\left(t_{1}\right)$ such that the external surfaces of $\mathcal{A}$ and $\mathcal{B}(t)$ do not have common points for all $t \in\left[t_{0}, t_{1}\right]$. We suppose that $h(\lambda, t)=0$ does not have four distinct negative roots and we will proof that there is a contradiction. From (Bhatia, 1997) we associate continuous roots to all continuous polynomials with complex coefficients. By theorem 2.2, $h(\lambda)=0$ has two negative roots which we denote by $\lambda_{3}(t)$ and $\lambda_{4}(t)$. The two other roots are denoted $\lambda_{1}(t)$ and $\lambda_{2}(t)$. For $\mathcal{B}\left(t_{0}\right), h(\lambda)=0$ has four negative roots and therefore $\lambda_{1}\left(t_{0}\right)$ and $\lambda_{2}\left(t_{0}\right)$ are negative.

If the roots change signs then:

- $\lambda_{1}(t)$ and $\lambda_{2}(t)$ change signs from negative to positive through 0 or $\infty$, without ever becoming imaginary;

- $\lambda_{1}(t)$ and $\lambda_{2}(t)$ are a pair of imaginary conjugate roots.

For the first case, if $h(\lambda)=0$ has a positive roots then the two ellipsoids are separated (Wang et al., 2001), which is impossible because for all $t \in\left[t_{0}, t_{1}\right]$ the ellipsoid $\mathcal{B}(t)$ is included in $\mathcal{A}$. From Equation (3) the leading and the last coefficients of $h(\lambda, t)=0$ are $-(a b c)^{-2}$ and $-r^{2}$, respectively. Thus, the product of the four roots of $h(\lambda, t)=0$ is $(a b c r)^{2}>0$.

In the second case, let $\lambda_{1}(t)$ and $\lambda_{2}(t)$ be a pair of imaginary conjugate roots. Let $l(\lambda, t)$ be the function such that $h(\lambda, t)=\alpha\left(\lambda-\lambda_{3}(t)\right)\left(\lambda-\lambda_{4}(t)\right) l(\lambda, t)$. Then, the equation $l(\lambda, t)=0$ has a negative discriminant $\Delta\left(t_{i}\right)$, and $\Delta\left(t_{i}\right)=0$ for some $t_{i} \in\left[t_{0}, t_{1}\right]$. For this $t_{i}$, we have $\lambda_{1}\left(t_{i}\right)=\lambda_{2}\left(t_{i}\right)$. In the first case $\lambda_{1}\left(t_{i}\right)$ and $\lambda_{2}\left(t_{i}\right)$ can not be positive roots. Then $\lambda_{1}\left(t_{i}\right)=\lambda_{2}\left(t_{i}\right)<0$. From lemma 2.4, we have that $\mathcal{A}$ and $\mathcal{B}\left(t_{i}\right)$ are tangent internally. This is impossible because the external surfaces of $\mathcal{A}$ and $\mathcal{B}(t)$ do not have common points for all $t \in\left[t_{0}, t_{1}\right]$. We therefore conclude that both $\lambda_{1}(t)$ and $\lambda_{2}(t)$ are negative for all $t \in\left[t_{0}, t_{1}\right]$, and hence $h(\lambda)=0$ has four negative roots.

Lemma 2.7: If the characteristic equation $h(\lambda)=0$ has four distinct negative roots, then either $\mathcal{A} \cap \mathcal{B}=\mathcal{A}$ or $\mathcal{B}$.

Proof: Suppose that $h(\lambda)=0$ has four distinct negative roots. Then one and only one of the following four situations holds:

(1) $\mathcal{A}$ and $\mathcal{B}$ are separated.

(2) The external surfaces of $\mathcal{A}$ and $\mathcal{B}$ have common points.

(3) $\mathcal{A}$ and $\mathcal{B}$ are tangent.

(4) $\mathcal{A} \cap \mathcal{B}=\mathcal{A}$ or $\mathcal{B}$.

The first case is impossible because if $\mathcal{A}$ and $\mathcal{B}$ are separated then by (Wang et al., 2001) $h(\lambda)=0$ has two distinct positive roots. If the external surfaces of $\mathcal{A}$ and $\mathcal{B}$ have common points which is not possible by lemma 2.5. If $\mathcal{A}$ and $\mathcal{B}$ are tangent then $h(\lambda)=0$ has positive or negative double roots which is a contradiction. Therefore, the only possible situation is $\mathcal{A} \cap \mathcal{B}=\mathcal{A}$ or $\mathcal{B}$.

Theorem 2.8: $\quad$ Let two ellipsoids $\mathcal{A}$ and $\mathcal{B}$ with the characteristic equation $h(\lambda)=0$.

(1) $\mathcal{A}$ and $\mathcal{B}$ touch each other internally at a single point, if and only if $h(\lambda)=0$ has a negative double root.

(2) $\mathcal{A} \cap \mathcal{B}=\mathcal{A}$ or $\mathcal{B}$, if and only if $h(\lambda)=0$ has four negative roots. 
Proof:

(1) For the first part of this theorem we have:

- "If": If $\mathcal{A}$ and $\mathcal{B}$ touch each other internally at a single point, then by lemma $2.3 h(\lambda)=0$ has a negative double root.

- "Only if": If $h(\lambda)=0$ has a negative double root, then by lemma $2.4 \mathcal{A}$ and $\mathcal{B}$ touch each other internally at a single point.

(2) For the second part:

- "If": If $\mathcal{A} \cap \mathcal{B}=\mathcal{A}$ or $\mathcal{B}$, then by lemma $2.6 h(\lambda)=0$ has four negative roots.

- "Only if": if $h(\lambda)=0$ has four negative roots, then by lemma 2.7 and lemma $2.4 \mathcal{A} \cap \mathcal{B}=\mathcal{A}$ or $\mathcal{B}$.

\section{Internal contact algorithm}

In the geometric potential method ( $\mathrm{Lin}$ and $\mathrm{Ng}$, 1995), one needs to solve the two nonlinear systems. Here we propose an iterative procedure that converges rapidly to the solution of such systems. The principle idea of the proposed algorithm is that it combines the algebraic condition of the contact of two ellipsoids with the geometric potential method (See Figure 3).

For convenience, let $f$ and $g$ be the real-valued functions defined on $\mathbb{R}^{4}$ by:

$$
\left\{\begin{array}{l}
f(\boldsymbol{x})=\boldsymbol{x}^{T} A \boldsymbol{x} \\
g(\boldsymbol{x})=\boldsymbol{x}^{T} B \boldsymbol{x}
\end{array}\right.
$$

such that the boundaries of the two ellipsoids $\mathcal{A}$ and $\mathcal{B}$ are defined by $f(\boldsymbol{x})=0$ and $g(\boldsymbol{x})=0$, respectively.

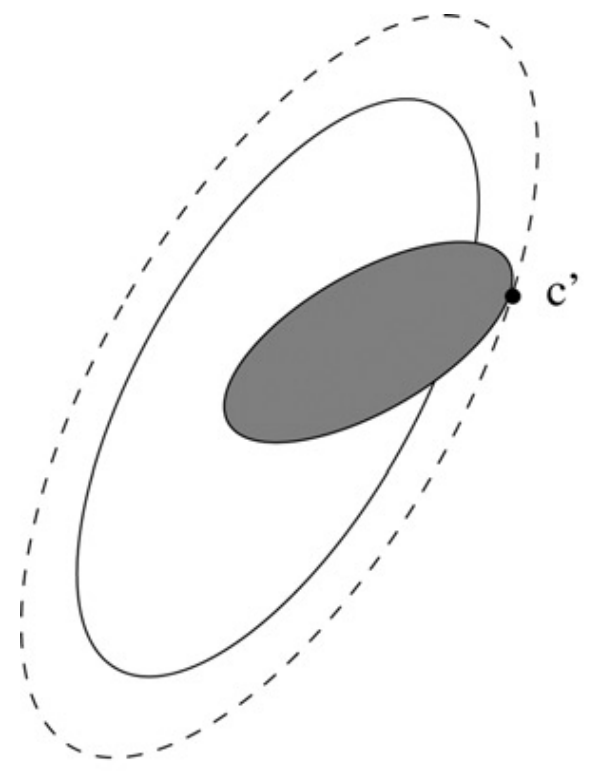

(a)

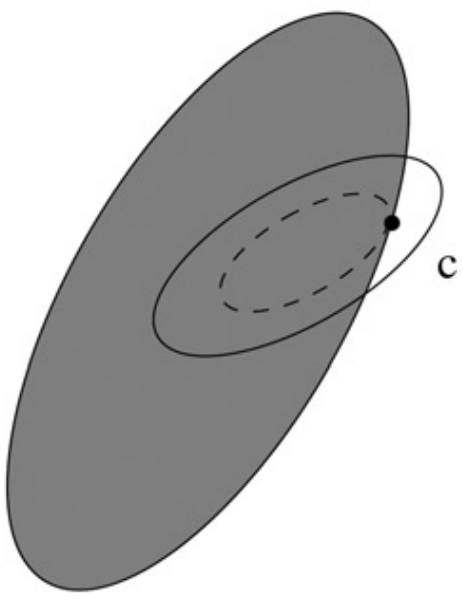

Figure 3.

Internal contact of two ellipsoids 
For each $e \in[-1,+\infty)$, the set of points $\boldsymbol{x}$ such that $f(\boldsymbol{x})=e$ is the boundary of an ellipsoid $\mathcal{A}_{e}$ that is similar to $\mathcal{A} \equiv \mathcal{A}_{0}$. Similarly, for each $e \in[-1,+\infty)$, the set of points $\boldsymbol{x}$ such that $g(\boldsymbol{x})=e$ is the boundary of an ellipsoid $\mathcal{B}_{e}$ that is similar to $\mathcal{B} \equiv \mathcal{B}_{0}$.

If $\mathcal{A}$ and $\mathcal{B}_{e}$ have a real touching point $\boldsymbol{x}_{0}$, then $\boldsymbol{x}_{0}$ is the eigenvector of $-A^{-1} B_{e}$ associated with the eigenvalue $\lambda_{0}$. Our procedure for the determination of the possible point of contact between the externally surface of $\mathcal{A}$ and the externally surface of $\mathcal{B}$ is first to check if a contact exists. This can be quickly checked by looking if $h(\lambda)=0$ does not have four distinct negative roots. If so then we proceed in finding the points $C$ and $C^{\prime}$.

The problem is formulated as

$$
\left\{\begin{array}{l}
\text { Find } \boldsymbol{x}_{0} \text { and } e \text { such that } \\
\mathcal{A} \text { and } \mathcal{B}_{e} \text { has one real touching point } \boldsymbol{x}_{0} .
\end{array}\right.
$$

The search for $\boldsymbol{x}_{0}$ and $e$ solving this problem is a delicate geometrical problem. Indeed, the search for a vector $\boldsymbol{x}_{0}$ in the intersection of the two ellipsoids is a nonlinear problem. However, if the two externally surface of $\mathcal{A}$ and $\mathcal{B}$ overlap then we are guaranteed that there exists $e$ such that $h_{e}(\lambda)$ has a negative double root. As stated earlier, the equation $\boldsymbol{x}^{T} B_{e} \boldsymbol{x}=e$ defines the boundary of a family of ellipsoids $\mathcal{B}_{e}$. Therefore, we conclude that $e \in[0, \alpha]$ where $\alpha<2$. Hence, Equation (10) can be reformulated as

$$
\left\{\begin{array}{l}
\text { Find } e \in[0, \alpha] \text { such that } \\
h_{e}(\lambda) \text { has a negative double root. }
\end{array}\right.
$$

Our algorithm is to start with $e_{0} \in I_{0}=\left[a_{0}, b_{0}\right]$, where $a_{0}=0$ and $b_{0}=\alpha$. Then for each iteration $k=1,2, \ldots$ we check whether $\mathcal{A} \cap \mathcal{B}_{e_{k-1}}=\mathcal{B}_{e_{k-1}}$ by looking at the roots of $h_{e_{k-1}}(\lambda)=0$. If so, then $I_{k}=\left[a_{k}, b_{k}\right]$ where $a_{k}=\left(a_{k-1}+b_{k-1}\right) / 2$ and $b_{k}=b_{k-1}$. Otherwise, we take $I_{k}=\left[a_{k}, b_{k}\right]$ where $a_{k}=a_{k-1}$ and $b_{k}=\left(a_{k-1}+b_{k-1}\right) / 2$. This process is repeated until $\left|e_{k+1}-e_{k}\right|$ falls below a prescribed small value $\epsilon$.

\section{Algorithm:}

(1) Initialization: test $=$ false, $e_{0}=0, a_{0}=0, b_{0}=\alpha, k=0$.

(2) While (test $==$ false) Do

(3) If $h_{e_{k}}(\lambda)=0$ has a negative double root $\lambda_{k}$, then

$(\lambda, e)=\left(\lambda_{k}, e_{\boldsymbol{k}}+\right)$, test $=$ true.

Go to step 8.

(4) If $h_{e_{h}}(\lambda)=0$ has four negative roots, then $\mathcal{A} \cap \mathcal{B}_{e_{k}}=\mathcal{B}_{e_{k}}$

Take $a_{k+1}=e_{k}, b_{k+1}=b_{k}$

(5) Else the externally surfaces of $\mathcal{A}$ and $\mathcal{B}_{e_{k}}$ overlap.

Take $a_{k+1}=a_{k}, b_{k+1}=e_{k}$.

(6) $e_{k+1}=\frac{a_{k+1}+b_{k+1}}{2}$.

$k=k+1$.

(7) If $\left|e_{k-1}-e_{k}\right|<\varepsilon$ then test $=$ true.

(8) End Do.

(9) Find $x_{0}$ solution of the linear system $\left(\lambda A+B_{e}\right) x=0$. 
Convergence of this iterative procedure is guaranteed by the fact that in each iteration the interval $I_{k}=\left[a_{k}, b_{k}\right]$ for the possible values of $e_{k}$ is cut in halves. Let $d_{k+1}=\left|e_{k+1}-e_{k}\right|$. Then, $d_{0}=\alpha$ and $d_{1}=\left|e_{1}-e_{0}\right|=\frac{\alpha}{2}<1$, and for each $k=1,2, \ldots$ we have $d_{k+1}=\frac{1}{2} d_{k}=\frac{\alpha}{2^{k}}$.

The final step in the above algorithm is of course to find the contact point $C=\left(x_{c}, y_{c}, z_{c}\right)$ obtained by finding the eigenvector $\boldsymbol{x}_{0}=\left(x_{c}, y_{c}, z_{c}, 1\right)$ associated with $\lambda$, which is equivalent to solve the following linear system $\left(\lambda A+B_{e}\right) \boldsymbol{x}=0$.

\section{Numerical simulations}

For the numerical simulation of granular materials presented here, 10,000 ellipsoidal particles are randomly placed inside a circular cylinder with radius $R=0.06 \mathrm{~m}$ and length $L=0.08 \mathrm{~m}$. Under the action of the gravity alone the particles are settled at the bottom part of the cylinder. After this initialization step, the cylinder is rotated around its axis of revolution with a constant angular velocity equal to 30 R.P.M. In Table I, we summarize the particle properties employed in this numerical simulation.

After few time steps, two motion zones appear: an internal zone that rotates almost as a rigid body, and an external zone characterized by a rapid flow. In Figure 4, we show two snapshots of the rotative cylinder at $t=1$ s and at $t=1.3 \mathrm{~s}$.

\section{Conclusion and future work}

The introduction of the algebraic condition of internal contact can be generalized for other quadratic forms like cylinder. However, the new algorithm for the calculation of the contact point introduces a new approach for the resolution of the contact problems, which can be useful for other convex forms.

\begin{tabular}{ll}
\hline Particle number & 10,000 \\
Particle semi-axes & $(2,3,2) \mathrm{mm}$ \\
Mass & $0.125 \mathrm{~g}$ \\
Angular velocity & $30 \mathrm{R} . \mathrm{P.M}$. \\
Normal stiffness coefficient & $3,000 \mathrm{~Pa}$ \\
Restitution coefficient & 0.7 \\
Friction coefficient & 0.4 \\
Ratio of tangential/normal stiffnesses & 0.7
\end{tabular}

Table I. Ellipsoidal particle data
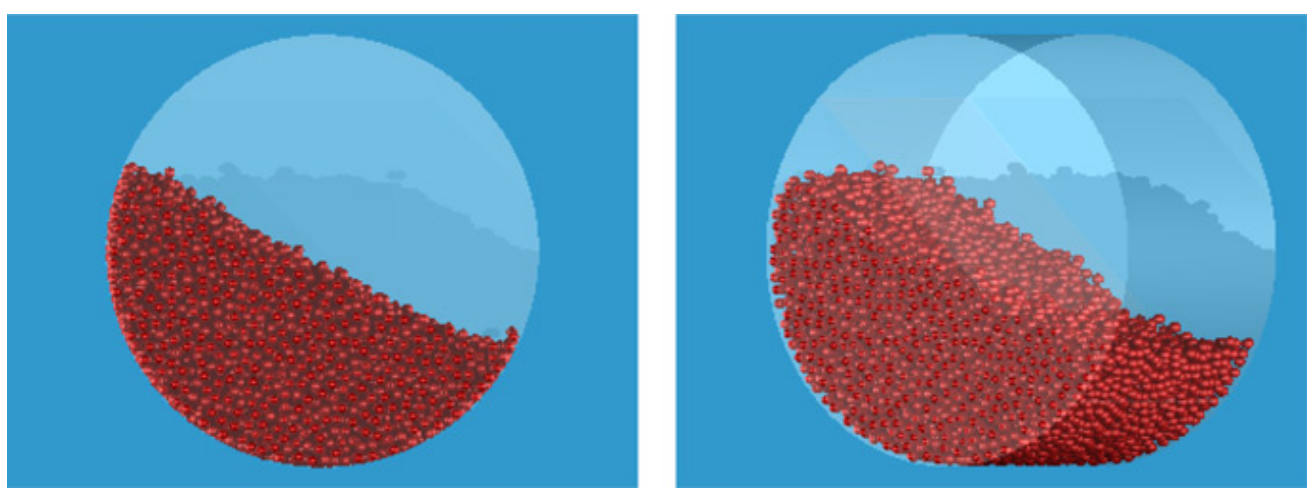

Figure 4. Snapshots of the rotative cylinder at $t=1 \mathrm{~s}$ and at $t=1.3 \mathrm{~s}$ 
It is clear that this new algorithm is efficient in the case of overlapping ellipsoids, and especially for the calculation of the contact point and the overlapping distance with a high degree of accuracy. The numerical behavior and the optimization of this new algorithm are also of importance, it will be the goal of future work.

\section{References}

Bhatia, R. (1997), Matrix Analysis, Graduate Textbook of Mathematics, Springer, New York, NY, p. 169.

Bromwich, T. (1906), "Quadratic forms and their classification by means of invariant-factors", Cambridge Tracts in Mathematics and Mathematical Physics, Vol. 3.

Farouki, R., Neff, C. and O'Connor, M. (1989), "Automatic parsing of degenerate quadric-surface intersections", ACM Transactions on Graphics, Vol. 8 No. 3, pp. 174-203.

Levin, J. (1979), "Mathematical models for determining the intersections of quadric surfaces", Computer Graphics and Image Processing, Vol. 11, pp. 73-87.

Lin, X. and Ng, T. (1995), "Contact detection algorithms for three-dimensional ellipsoids in discrete element modeling", International Journal for Numerical and Analytical Methods in Geomechanics, Vol. 19, pp. 653-9.

Perram, J. and Wertheim, M.S. (1985), "Statistical mechanics of hard ellipsoids. I: Overlap algorithm and the contact function", Journal of Computational Physics, Vol. 58, pp. 409-16.

Perram, J., Rasmussen, J., Prastaard, E. and Lebowitz, J. (1996), "Ellipsoids contact potential: theory and relation to overlap potentials", Physical Review E, Vol. 54 No. 6, pp. 6565-72.

Sommerville, D. (1947), Analytical Geometry of Three Dimensions, Cambridge University Press, Cambridge.

Trabelsi, B. and Moakher, M. (2006), "An efficient algorithm for discrete element simulation of granular mixing of ellipsoidal grains", Proceedings of the First Euromediterranean Symposium on Advances in Geomaterials and Structures, Tunisia, pp. 77-81.

Wang, W., Wang, J.Y. and Kim, M.-S. (2001), "An algebraic condition on the separation of two ellipsoids”, Computer Aided Geometric Design, Vol. 18 No. 6, pp. 531-9.

\section{Corresponding author}

Ibrahim Trabelsi can be contacted at: brahim.trabelsi@lamsin.rnu.tn 